\title{
Bifurcation Control of a Delayed Fractional Mosaic Disease Model for Jatropha curcas with Farming Awareness
}

\author{
Shouzong Liu $\left(\mathbb{D}\right.$, Mingzhan Huang $\mathbb{D}^{D}$, and Juan Wang \\ College of Mathematics and Statistics, Xinyang Normal University, Xinyang 464000, China \\ Correspondence should be addressed to Mingzhan Huang; huangmingzhan@163.com
}

Received 1 March 2020; Revised 13 May 2020; Accepted 21 May 2020; Published 27 June 2020

Academic Editor: Mohamed Boutayeb

Copyright ( 92020 Shouzong Liu et al. This is an open access article distributed under the Creative Commons Attribution License, which permits unrestricted use, distribution, and reproduction in any medium, provided the original work is properly cited.

\begin{abstract}
In this paper, the bifurcation control of a fractional-order mosaic virus infection model for Jatropha curcas with farming awareness and an execution delay is investigated. By analyzing the associated characteristic equation, Hopf bifurcation induced by the execution delay is studied for the uncontrolled system. Then, a time-delayed controller is introduced to control the occurrence of Hopf bifurcation. Our study implies that bifurcation dynamics is significantly affected by the change of the fractional order, the feedback gain and the extended feedback delay provided that the other parameters are fixed. A series of numerical simulations is performed, which not only verifies our theoretical results but also reveals some specific features. Numerically, we find that the Hopf bifurcation gradually occurs in advance with the increase of the fractional order, and there exist extreme points for the feedback gain and the extended feedback delay which can minimize the bifurcation value.
\end{abstract}

\section{Introduction}

Jatropha curcas is a plant that is commonly seen in tropical and subtropical areas. It can grow well in marginal and poor soil, grows fast, and lives relatively long. Meanwhile, it produces seeds with an oil content of about $27 \%$ to $40 \%$. The oil burns with clear smoke-free flame and can be used to produce biodiesel fuel [1]. Therefore, as one of the most suitable alternative renewable energy resources, it is widely planted in many countries $[2,3]$. However, many studies showed that Jatropha plants are hosts of mosaic virus which is a main cause of the occurrence of viruses in Jatropha curcas $[4,5]$. Early reports about virus infections of Jatropha plants indicated that the occurrence of Cassava mosaic virus in India causes a high disease incidence from 25 to $47 \%$. This constitutes a major obstacle to the large scale planting of Jatropha [6]. When the Jatropha plant is infected with mosaic virus, its fruit will be attacked and the yield and the quality of oil may be severely affected.

Awareness of the disease is really necessary for farmers who plant Jatropha curcas because they can take action to prevent or mitigate the problem in time. Effective knowledge can provide proper control measures when the virus infection outbreaks. It is advisable to educate the Jatropha curcas growers with agricultural information about agronomic practices and plant protection measures [7]. Common platforms for mass media such as newspapers, magazines, radio, television, and the Internet are main ways to propagandize correct and relevant information about this crop and its disease. There are also new technologies adopted in agricultural awareness programs $[8,9]$. These are very important for the grower who is engaged in the crop production. For example, Yang et al. exploited the levels of knowledge and awareness of the side effects of insecticides to the environment and human health in regions with different farming modes and found that farmers can improve their agronomic practice to reduce environmental hazard and protect human health after properly raising awareness [10]. Le Bellec et al. studied the collaboration mechanism of growers, researchers, and other factors which can alleviate the problem we face in crop management [11]. Basir et al. proposed a mathematical model to investigate the impact of awareness programs on the protection of the Jatropha curcas plant against possible epidemic spread of the mosaic disease [3].

Generally speaking, when Jatropha curcas growers realize the harm of virus infection, they need to take some time before they can take appropriate measures to prevent or 
reduce the occurrence of this disease. So, it is more plausible to consider an execution time delay when building such kind of virus infection model. Basir et al. analyzed how execution time delay in accepting the awareness campaign can affect the ultimate dynamics of the mosaic disease [2], while the authors in [3] investigated the Hopf bifurcation phenomenon of an epidemic model with awareness programs due to the execution time delay. Basir et al. [12] proposed a mathematical model and analyzed the effect of awareness programs on the control of pest in agricultural practice. However, these studies are all aimed at integer-order models. In this work, we further analyze the stability and Hopf bifurcation of a fractional-order virus infection model with execution delay of awareness programs.

In recent decades, fractional calculus theory has been widely applied in biology, optical and thermal system, materials science, electromagnetic field theory, mechanical mechanics, and so on $[13,14]$. It has been found that fractional calculus can accurately describe the rules and development process of some phenomena in natural science. Furthermore, it is found that fractional-order differential system has the advantages of simple modeling, clear parameter meaning, and accurate description for some materials and processes with memory and genetic characteristics [15-17]. Hence, fractional calculus has become a new mathematical tool favored by researchers, and more and more study of practical problems introduces the theory of fractional calculus and remarkable achievements have been made [18-30].

For a given nonlinear system, a controller aiming at modifying the bifurcation behavior is usually designed, so as to achieve some desirable dynamical behaviors, which is often called bifurcation control [31,32]. According to the different objectives of bifurcation control, different control strategies are formulated, such as PD control, time-delayed feedback control, and hybrid control. For example, Xiao et al. [22] adopted a PD control method to control Hopf bifurcations in delayed fractional-order small-world networks, while $\mathrm{Lu}$ et al. [32] analysed the stability and bifurcation of a fractional-order single-gene regulatory model under a PD control law. Xu et al. [28] applied two timedelayed feedback controllers to regulate a fractional-order chaotic Genesio-Tesi model, and Huang et al. [33] designed a hybrid controller for the first time to control the Hopf bifurcation of a network model.

As an effective control scheme, time-delayed feedback control was proposed by constructing a control force using the difference between the current state and its delay value, i.e., $x(t)-x(t-\delta)$, and once the system reaches a stable state, the control force vanishes [34]. The main advantage of this scheme is that it is noninvasive and it does not require a reference system because the control is generated from the information of the system itself. Besides, this scheme can be easily implemented in the actual system. Hence, in recent years, the bifurcation controls of many dynamic systems in many fields are studied by introducing time-delayed feedback controllers.

In this paper, delayed fractional mosaic disease models for Jatropha curcas with farming awareness are proposed.
We will study the stability and bifurcation of the system and discuss the bifurcation control by introducing a timedelayed feedback controller.

This paper is organized as follows. In Section 2, some basic materials regarding fractional calculus are presented. The delayed fractional mosaic disease models with and without control are proposed in Section 3. In Section 4, detailed analysis of bifurcation phenomena for the two systems is carried out. Series of numerical simulations are carried out in Section 5, which not only confirm the theoretical results we have obtained but also are complementary to those results with specific features. The paper ends with a brief conclusion.

\section{Preliminaries}

In this section, we will briefly introduce some notations and definitions about fractional calculus theory, which will be useful in the following discussion. There are several kinds of definitions of fractional derivatives proposed in previous research, such as Riemann-Liouville fractional derivative, Grünwald-Letnikov fractional derivative, Caputo fractional derivative, Weyl fractional derivative, and Marchaud fractional derivative [35]. It is important to note that Caputo fractional derivative has the advantage of requiring the initial conditions to be easily derived from the controlled system, which make it easier to apply to practical problems [32]. Therefore, in this paper, we only study Caputo fractional derivative.

Definition 1 (see [36]). The Caputo fractional-order derivative with fractional order $\alpha$ for a continuous function $p(t): R_{+} \longrightarrow R^{n}$ is defined by

$$
{ }_{C} D_{t_{0}, t}^{\alpha} p(t)=\frac{1}{\Gamma(m-\alpha)} \int_{t_{0}}^{t}(t-\tau)^{m-\alpha-1} p^{(m)}(\tau) \mathrm{d} \tau,
$$

where $0 \leq m-1 \leq \alpha<m, m \in Z^{+}$and $\Gamma(\cdot)$ is the Gamma function. The constant $\alpha$ is the value of the fractional order.

$$
\begin{aligned}
& \text { Especially, } \\
& { }_{C} D_{t_{0}, t}^{\alpha} p(t)=(1 / \Gamma(m-\alpha)) \int_{t_{0}}^{t}(t-\tau)^{\alpha} p^{\prime}(\tau) \mathrm{d} \tau .
\end{aligned}
$$

Remark 1. Our work is based on the Caputo derivative, and for convenience, in this work, we denote the Caputo fractional-order derivative operator ${ }_{C} D_{t_{0}, t}^{\alpha} p(t)$ by $D^{\alpha} p(t)$ and suppose that $\alpha \in[0,1]$.

Definition 2 (see [37]). Consider the following $n$-dimension fractional-order system:

$$
D^{\alpha} x(t)=f(x(t))
$$

where $x(t)=\left(x_{1}(t), x_{2}(t), \ldots, x_{n}(t)\right)$ and $f(t)=\left(f_{1}(x\right.$ $\left.(t)), f_{2}(x(t)), \ldots, f_{n}(x(t))\right)$. The equilibrium point $x^{*}=$ $\left(x_{1}^{*}, x_{2}^{*}, \ldots, x_{n}^{*}\right)$ is defined by the algebraic equation $f_{i}\left(x_{1}, x_{2}, \ldots, x_{n}\right)=0, i=1,2, \ldots, n$.

The stability of the solution of the above $n$-dimension system is defined in a lot of literature studies. For more details, readers can refer to [38]. 


\section{Model Formulation}

The authors in [2] studied an integral order model of virus infection for Jatropha curcas with farming awareness. An execution delay is incorporated, which measures from the moment that the relevant information is available to the moment that farmers take action with these knowledge. They mainly explored the impact of the time delay on the dynamical behaviors.

Motivated by the work in [2], in this paper, we consider the virus infection model with fractional-order and the execution delay; then, we propose the following delayed fractional-order virus infectious model:

$$
\left\{\begin{array}{l}
D^{\alpha} P_{S}(t)=r P_{S}(t)\left(1-\frac{P_{S}(t)+P_{I}(t)}{K}\right)-a P_{S}(t) V(t), \\
D^{\alpha} P_{I}(t)=a P_{S}(t) V(t)-m P_{I}(t)-n M(t-\tau) P_{I}(t), \\
D^{\alpha} V(t)=b P_{I}(t)-c V(t)-\mathrm{d} M(t-\tau) V(t), \\
D^{\alpha} M(t)=\gamma+\beta P_{I}(t)-\eta M(t),
\end{array}\right.
$$

with $P_{S}(0) \geq 0, P_{I}(0) \geq 0, V(0) \geq 0, M(0) \geq 0$, and $0<\alpha \leq 1$.

The interpretations of the variables and parameters are listed in Table 1.
TABLE 1: Descriptions of parameters in (3).

\begin{tabular}{lr}
\hline Parameter & Description \\
\hline$P_{S}(t)$ & The healthy plant biomass at time $t$ \\
$P_{I}(t)$ & The infected plant biomass at time $t$ \\
$V(t)$ & The number of infected vectors at time $t$ \\
$M(t)$ & The density of aware people at time $t$ \\
$r$ & The intrinsic growth rate of healthy plants \\
$K$ & The carrying capacity of healthy plants \\
$a$ & The contact rate between healthy plants and infected \\
$m$ & The disease-induced death rate of infected plants \\
$n$ & The harvesting rate of infected plants by aware people \\
$b$ & The appearance rate of infected vector \\
$c$ & The death rate of infected vectors \\
$d$ & The removal rate of vectors by aware \\
$\gamma$ & The rate of global awareness \\
$\beta$ & The recruitment rate of aware people \\
$\eta$ & The fading rate of awareness \\
$\tau$ & The execution delay
\end{tabular}

To control the bifurcation caused by the execution delay, we introduce a time-delayed feedback controller as follows:

$$
k\left[P_{S}(t)-P_{S}(t-\delta)\right] .
$$

Then, we gain the following controlled system:

$$
\left\{\begin{array}{l}
D^{\alpha} P_{S}(t)=r P_{S}(t)\left(1-\frac{P_{S}(t)+P_{I}(t)}{K}\right)-a P_{S}(t) V(t)+k\left[P_{S}(t)-P_{S}(t-\delta)\right] \\
D^{\alpha} P_{I}(t)=a P_{S}(t) V(t)-m P_{I}(t)-n M(t-\tau) P_{I}(t), \\
D^{\alpha} V(t)=b P_{I}(t)-c V(t)-d M(t-\tau) V(t), \\
D^{\alpha} M(t)=\gamma+\beta P_{I}(t)-\eta M(t) .
\end{array}\right.
$$

Remark 2. $k<0$ is the negative feedback gain and $\delta>0$ stands for the delay of feedback control. In the field of ecological control, with the aim of enhancing the stability performance, the farmers may harvest or transplant some plants on the basis of past data $\left(P_{S}(t-\delta)\right)$.

We will study the existence of Hopf bifurcation and explore the impact of time delay, the fractional order, the feedback gain, and the feedback delay on the occurrence of the bifurcation.

\section{Main Results}

In this section, we firstly study the stability of the coexistence equilibrium and the Hopf bifurcation caused by the time delay for the uncontrolled system (3). Then, the impact of the fractional order and the feedback control on the occurrence of the bifurcation is investigated for the controlled system (5).

The system (3) has three equilibria: the plant-vector-free equilibrium $E_{1}=(0,0,0,(\gamma / \eta))$, the disease-free equilibrium $E_{2}=(K, 0,0,(\gamma / \eta))$, and the coexistence equilibrium $E^{*}=\left(P_{S}^{*}, P_{I}^{*}, V^{*}, M^{*}\right)$. By direct calculation, we can easily obtain

$$
\begin{aligned}
P_{S}^{*} & =\frac{\left(m+n M^{*}\right)\left(c+\mathrm{d} M^{*}\right)}{a b}, \\
P_{I}^{*} & =\frac{\eta M^{*}-\gamma}{\beta}, \\
V^{*} & =\frac{b P_{I}^{*}}{c+\mathrm{d} M^{*}},
\end{aligned}
$$

and $M^{*}$ is the positive root of the following equation: 


$$
f(x)=a_{1} x^{3}+a_{2} x^{2}+a_{3} x+a_{4}=0,
$$

where

$$
\begin{aligned}
& a_{1}=\beta r n d^{2}>0, \\
& a_{2}=r d(a d \eta+2 c \beta n+\beta d m)>0, \\
& a_{3}=\beta r\left(2 c d m+c^{2} n-k a b d\right)+a b[r(c \eta-d \gamma)+K a \eta], \\
& a_{4} r \beta c(m c-K a b)-a b \gamma(r c+K a) .
\end{aligned}
$$

Obviously, if $a_{4}<0$, the above cubic polynomial equation has a unique positive root which is denoted by $x=M^{*}$. Then, we give the following result.

\section{Proposition 1. Assume that}

$$
\left(H_{1}\right): a_{4}<0 \text { and } \eta M^{*}>\gamma \text {. }
$$

Then, system (3) has a unique coexistence equilibrium $E^{*}=\left(P_{S}^{*}, P_{I}^{*}, V^{*}, M^{*}\right)$.

For convenience, let

$$
\begin{aligned}
& m_{1}=m+n M^{*}, \\
& m_{2}=c+\mathrm{d} M^{*}, \\
& m_{3}=m_{1}+m_{2}, \\
& m_{4}=m+n M^{*}+a V^{*}, \\
& m_{5}=m+n M^{*}+\frac{r}{K} P_{S}^{*}, \\
& m_{6}=m_{1} m_{2},
\end{aligned}
$$

and these denotations will be used in the following discussion.

4.1. Bifurcation Analysis of the Uncontrolled System (3). In this section, by choosing the execution delay $\tau$ as a bifurcation parameter, we analyse the existence of Hopf bifurcation and the critical value of the time delay at which a Hopf bifurcation occurs is obtained.

By linearizing (3) at $E^{*}$, we get the following Jacobian matrix:

$$
J\left(E^{*}\right)=\left(\begin{array}{cccc}
-\frac{r}{K} P_{S}^{*} & -\frac{r}{K} P_{S}^{*} & -a P_{S}^{*} & 0 \\
a V^{*} & -m-n M^{*} & a P_{S}^{*} & -n P_{I}^{*} e^{-s \tau} \\
0 & b & -c-\mathrm{d} M^{*} & -\mathrm{d} V^{*} e^{-s \tau} \\
0 & \beta & 0 & -\eta
\end{array}\right) .
$$

Thus, we have the following characteristic equation:

$$
\left|\begin{array}{cccc}
s^{\alpha}+\frac{r}{K} P_{S}^{*} & \frac{r}{K} P_{S}^{*} & a P_{S}^{*} & 0 \\
-a V^{*} & s^{\alpha}+m+n M^{*} & -a P_{S}^{*} & n P_{I}^{*} e^{-s \tau} \\
0 & -b & s^{\alpha}+c+\mathrm{d} M^{*} & \mathrm{~d} V^{*} e^{-s \tau} \\
0 & -\beta & 0 & s^{\alpha}+\eta
\end{array}\right|=0 .
$$

Through simplification, we can obtain $s^{4 \alpha}+A_{1} s^{3 \alpha}+A_{2} s^{2 \alpha}+A_{3} s^{\alpha}+A_{4}+\left(A_{5} s^{2 \alpha}+A_{6} s^{\alpha}+A_{7}\right) e^{-s \tau}=0$,

where

$$
\begin{aligned}
A_{1}= & \eta+m_{2}+m_{5}, \\
A_{2}= & m_{6}+\frac{r}{K} P_{S}^{*}\left(m_{2}+m_{4}\right)-a b P_{S}^{*}+\eta\left(m_{2}+m_{5}\right), \\
A_{3}= & m_{2} m_{4} \frac{r}{K} P_{S}^{*}-a b P_{S}^{*}\left(\frac{r}{K} P_{S}^{*}-a V^{*}\right) \\
& +\eta\left(m_{2} m_{5}+m_{4} \frac{r}{K} P_{S}^{*}-a b P_{S}^{*}\right), \\
A_{4}= & \eta\left[m_{2} m_{4} \frac{r}{K} P_{S}^{*}-a b P_{S}^{*}\left(\frac{r}{K} P_{S}^{*}-a V^{*}\right)\right], \\
A_{5}= & \beta n P_{I}^{*}, \\
A_{6}= & \beta\left[n P_{I}^{*}\left(m_{2}+\frac{r}{K} P_{S}^{*}\right)+a d P_{S}^{*} V^{*}\right], \\
A_{7}= & \beta P_{S}^{*}\left[m_{2} n \frac{r}{K} P_{I}^{*}+a d V^{*}\left(\frac{r}{K} P_{S}^{*}-a V^{*}\right)\right] .
\end{aligned}
$$

Suppose that $\tau=0$, then equation (13) becomes $s^{4 \alpha}+A_{1} s^{3 \alpha}+\left(A_{2}+A_{5}\right) s^{2 \alpha}+\left(A_{3}+A_{6}\right) s^{\alpha}+A_{4}+A_{7}=0$.

Assume that

$$
\begin{aligned}
\left(H_{2}\right): A_{4}+A_{7} & >0, \\
A_{1}\left(A_{2}+A_{5}\right)-\left(A_{3}+A_{6}\right) & >0, \\
{\left[A_{1}\left(A_{2}+A_{5}\right)-\left(A_{3}+A_{6}\right)\right]\left(A_{3}+A_{6}\right)-A_{1}^{2}\left(A_{4}+A_{7}\right) } & >0 .
\end{aligned}
$$

Then, by the Routh-Hurwitz criterion, we have the following result.

Proposition 2. For $\tau=0$, if assumptions $\left(H_{1}\right)$ and $\left(H_{2}\right)$ are satisfied, then the roots of (15) are real and negative. Therefore, the coexistence equilibrium $E^{*}$ is locally asymptotically stable.

To study the phenomenon of Hopf bifurcation, we assume that $s=\omega i(\omega>0)$ is a root of (13), then, we can have 


$$
\begin{aligned}
& \omega^{4 \alpha}(\cos 2 \alpha \pi+i 2 \sin \alpha \pi)+A_{1} \omega^{3 \alpha}\left(\cos \frac{3 \alpha \pi}{2}+i \sin \frac{3 \alpha \pi}{2}\right)+A_{2} \omega^{2 \alpha}(\cos \alpha \pi+i \sin \alpha \pi) \\
& +A_{3} \omega^{\alpha}\left(\cos \frac{\alpha \pi}{2}+i \sin \frac{\alpha \pi}{2}\right)+A_{4}+\left[A_{5} \omega^{2 \alpha}(\cos \alpha \pi+i \sin \alpha \pi)\right. \\
& \left.+A_{6} \omega^{\alpha}\left(\cos \frac{\alpha \pi}{2}+i \sin \frac{\alpha \pi}{2}\right)+A_{7}\right](\cos \omega \tau-i \sin \omega \tau)=0 .
\end{aligned}
$$

Separating the real and imaginary parts, we obtain

$$
\left\{\begin{array}{l}
C_{1} \cos \omega \tau+C_{2} \sin \omega \tau=-C_{3}, \\
C_{2} \cos \omega \tau-C_{1} \sin \omega \tau=-C_{4},
\end{array}\right.
$$

where

$$
\begin{aligned}
C_{1}= & A_{5} \omega^{2 \alpha} \cos \alpha \pi+A_{6} \omega^{\alpha} \cos \frac{\alpha \pi}{2}+A_{7}, \\
C_{2}= & A_{5} \omega^{2 \alpha} \sin \alpha \pi+A_{6} \omega^{\alpha} \sin \frac{\alpha \pi}{2} \\
C_{3}= & \omega^{4 \alpha} \cos 2 \alpha \pi+A_{1} \omega^{3 \alpha} \cos \frac{3 \alpha \pi}{2}+A_{2} \omega^{2 \alpha} \cos \alpha \pi \\
& +A_{3} \omega^{\alpha} \cos \frac{\alpha \pi}{2}+A_{4}, \\
C_{4}= & \omega^{4 \alpha} \sin 2 \alpha \pi+A_{1} \omega^{3 \alpha} \sin \frac{3 \alpha \pi}{2}+A_{2} \omega^{2 \alpha} \sin \alpha \pi \\
& +A_{3} \omega^{\alpha} \sin \frac{\alpha \pi}{2} .
\end{aligned}
$$

It follows from (18) that

$$
\left\{\begin{array}{l}
\sin \omega \tau=\frac{C_{1} C_{4}-C_{2} C_{3}}{C_{1}^{2}+C_{2}^{2}}, \\
\cos \omega \tau=-\frac{C_{1} C_{3}+C_{2} C_{4}}{C_{1}^{2}+C_{2}^{2}} .
\end{array}\right.
$$

Since $\sin ^{2} \omega \tau+\cos ^{2} \omega \tau=1$, we can obtain

$$
C_{3}^{2}+C_{4}^{2}=C_{1}^{2}+C_{2}^{2}
$$

By simple calculation, we deduce

$$
\begin{aligned}
\lambda^{8} & +2 A_{1} \cos \frac{\alpha \pi}{2} \lambda^{7}+\left(A_{1}^{2}+2 A_{2} \cos \alpha \pi\right) \lambda^{6}+\left(2 A_{3} \cos \frac{3 \alpha \pi}{2}+2 A_{1} A_{2} \cos \frac{\alpha \pi}{2}\right) \lambda^{5} \\
& +\left(A_{2}^{2}+2 A_{1} A_{3} \cos \alpha \pi+2 A_{4} \cos 2 \alpha \pi-A_{5}^{2}\right) \lambda^{4}+\left(2\left(A_{1} A_{3}-A_{5} A_{6}\right) \cos \frac{\alpha \pi}{2}\right. \\
& \left.+2 A_{1} A_{4} \cos \frac{3 \alpha \pi}{2}\right) \lambda^{3}+\left(A_{3}^{2}+2\left(A_{2} A_{4}-A_{5} A_{7}\right) \cos \alpha \pi-A_{6}^{2}\right) \lambda^{2} \\
& +\left(2\left(A_{3} A_{4}-A_{6} A_{7}\right) \cos \frac{\alpha \pi}{2}\right) \lambda+A_{4}^{2}-A_{7}^{2}=0
\end{aligned}
$$

where $\lambda=\omega^{\alpha}$.

Assume that

$$
\left(H_{3}\right): A_{4}^{2}-A_{7}^{2}<0 .
$$

Then, (18) has at least one positive real root $\omega_{0}$. Denote $\tau^{(i)}=\frac{1}{\omega_{0}}\left[\arccos \frac{C_{1} C_{3}+C_{2} C_{4}}{C_{1}^{2}+C_{2}^{2}}+2 i \pi\right], \quad i=0,1,2, \ldots$

Define

$$
\tau^{0}=\min \left\{\tau^{(i)}\right\}, \quad i=0,1,2, \ldots
$$

To derive conditions for the existence of Hopf bifurcation, we make the following hypothesis:

$$
\left(H_{4}\right): \frac{\Phi_{1} \Psi_{1}+\Phi_{2} \Psi_{2}}{\Psi_{1}^{2}+\Psi_{2}^{2}} \neq 0
$$

where $\Phi_{1}, \Phi_{2}, \Psi_{1}$, and $\Psi_{2}$ are defined by equation (30).

Lemma 1. Let $s(\tau)=\varphi(\tau)+i \omega(\tau)$ be a root of the characteristic equation (13) near $\tau=\tau^{(i)}$ meeting $\varphi\left(\tau^{(i)}\right)=$ $0, \omega\left(\tau^{(i)}\right)=\omega_{0}$; then, the transversality condition

$$
\left.\operatorname{Re}\left[\frac{\mathrm{d} s}{\mathrm{~d} \tau}\right]\right|_{\left(\tau=\tau^{0}, \omega=\omega_{0}\right)} \neq 0,
$$

holds.

Proof. Differentiating both sides of (13) with respect to $\tau$, we obtain 


$$
\frac{\mathrm{d} s}{\mathrm{~d} \tau}=\frac{\Phi(s)}{\Psi(s)},
$$

where $\Phi(s)=\left(A_{5} s^{2 \alpha}+A_{6} s^{\alpha}+A_{7}\right) s e^{-s \tau}$ and

$$
\begin{aligned}
& \Psi(s)=4 \alpha s^{4 \alpha-1}+3 \alpha A_{1} s^{3 \alpha-1}+2 \alpha A_{2} s^{2 \alpha-1}+\alpha A_{3} s^{\alpha-1} \\
& +\left[2 \alpha A_{5} s^{2 \alpha-1}+\alpha A_{6} s^{\alpha-1}-\left(A_{5} s^{2 \alpha}+A_{6} s^{\alpha}+A_{7}\right) \tau\right] e^{-s \tau} .
\end{aligned}
$$

Let

$$
\begin{aligned}
& \left.\Phi\left(\omega_{0} i\right)\right|_{\tau=\tau^{0}}=\Phi_{1}+i \Phi_{2}, \\
& \left.\Psi\left(\omega_{0} i\right)\right|_{\tau=\tau^{0}}=\Psi_{1}+i \Psi_{2},
\end{aligned}
$$

where

$$
\begin{aligned}
& \Phi_{1}=n_{1} \sin \omega_{0} \tau^{0}-n_{2} \cos \omega_{0} \tau^{0}, \\
& \Phi_{2}=n_{1} \cos \omega_{0} \tau^{0}+n_{2} \sin \omega_{0} \tau^{0}, \\
& \Psi_{1}=n_{3}+n_{5} \cos \omega_{0} \tau^{0}+n_{6} \sin \omega_{0} \tau^{0}, \\
& \Psi_{2}=n_{4}-n_{5} \sin \omega_{0} \tau^{0}+n_{6} \cos \omega_{0} \tau^{0},
\end{aligned}
$$

4.2. Bifurcation Control of System (5). In this section, we exploit the bifurcation control problem of system (5) through the time-delayed feedback controller.

Analogously, by linearizing (5) at $E^{*}$, we get the Jacobian matrix:

$$
J\left(E^{*}\right)=\left(\begin{array}{cccc}
-\frac{r}{K} P_{S}^{*}+k\left(1-e^{-s \delta}\right) & -\frac{r}{K} P_{S}^{*} & -a P_{S}^{*} & 0 \\
a V^{*} & -m-n M^{*} & a P_{S}^{*} & -n P_{I}^{*} e^{-s \tau} \\
0 & b & -c-\mathrm{d} M^{*} & -\mathrm{d} V^{*} e^{-s \tau} \\
0 & \beta & 0 & -\eta
\end{array}\right) \text {, }
$$

and the corresponding characteristic equation:

where

$s^{4 \alpha}+B_{1} s^{3 \alpha}+B_{2} s^{2 \alpha}+B_{3} s^{\alpha}+B_{4}+\left(B_{5} s^{2 \alpha}+B_{6} s^{\alpha}+B_{7}\right) e^{-s \tau}=0$,

$$
\begin{aligned}
& B_{1}=A_{1}-k\left(1-e^{-s \delta}\right), \\
& B_{2}=A_{2}-\left(m_{3}+\eta\right) k\left(1-e^{-s \delta}\right), \\
& B_{3}=A_{3}-\left[m_{6}+m_{3} \eta-a b P_{S}^{*}\right] k\left(1-e^{-s \delta}\right), \\
& B_{4}=A_{4}-\eta\left[m_{6}-a b P_{S}^{*}\right] k\left(1-e^{-s \delta}\right), \\
& B_{5}=A_{5}, \\
& B_{6}=A_{6}-\beta n P_{I}^{*} k\left(1-e^{-s \delta}\right), \\
& B_{7}=A_{7}-\left(m_{2} n P_{I}^{*}+a d P_{S}^{*} V^{*}\right) \beta k\left(1-e^{-s \delta}\right) .
\end{aligned}
$$

Assume that $s=\omega i(\omega>0)$ is a root of (11); then, we obtain 


$$
\begin{aligned}
& \omega^{4 \alpha}(\cos 2 \alpha \pi+i \sin 2 \alpha \pi)+\left[A_{1}-k(1-\cos \delta \omega+i \sin \delta \omega)\right] \omega^{3 \alpha}\left(\cos \frac{3 \alpha \pi}{2}+i \sin \frac{3 \alpha \pi}{2}\right) \\
& +\left[A_{2}-\left(m_{3}+\eta\right) k(1-\cos \delta \omega)-i\left(m_{3}+\eta\right) k \sin \delta \omega\right] \omega^{2 \alpha}(\cos \alpha \pi+i \sin \alpha \pi) \\
& +\left[A_{3}-\left(m_{6}+m_{3} \eta-a b P_{S}^{*}\right) k(1-\cos \delta \omega)-i\left(m_{6}+m_{3} \eta-a b P_{S}^{*}\right) k \sin \delta \omega\right] \omega^{\alpha} \\
& \times\left(\cos \frac{\alpha \pi}{2}+i \sin \frac{\alpha \pi}{2}\right)+A_{4}-\eta\left(m_{6}-a b P_{S}^{*}\right) k(1-\cos \delta \omega-i \sin \delta \omega) \\
& +\left\{A_{5} \omega^{2 \alpha}(\cos \alpha \pi+i \sin \alpha \pi)+\left[A_{6}-\beta n P_{I}^{*} k(1-\cos \delta \omega)-i \beta n P_{I}^{*} k \sin \delta \omega\right]\right. \\
& \times \omega^{\alpha}\left(\cos \frac{\alpha \pi}{2}+i \sin \frac{\alpha \pi}{2}\right)+\left[A_{7}-\left(m_{2} n P_{I}^{*}+a d P_{S}^{*} V^{*}\right) \beta k(1-\cos \delta \omega)\right. \\
& \left.\left.-i\left(m_{2} n P_{I}^{*}+a d P_{S}^{*} V^{*}\right) \beta k \sin \delta \omega\right]\right\}(\cos \omega \tau-i \sin \omega \tau)=0 .
\end{aligned}
$$

Separating the real and imaginary parts, we obtain

$$
\left\{\begin{array}{l}
D_{1} \cos \omega \tau+D_{2} \sin \omega \tau=-D_{3} \\
D_{2} \cos \omega \tau-D_{1} \sin \omega \tau=-D_{4}
\end{array}\right.
$$

where

$$
\begin{aligned}
D_{1}= & \varphi_{1} \cos \alpha \pi+\varphi_{2} \cos \frac{\alpha \pi}{2}+\varphi_{3} \sin \frac{\alpha \pi}{2}+\varphi_{4}, \\
D_{2}= & \varphi_{1} \sin \alpha \pi+\varphi_{2} \sin \frac{\alpha \pi}{2}-\varphi_{3} \cos \frac{\alpha \pi}{2}-\varphi_{5}, \\
D_{3}= & \omega^{4 \alpha} \cos 2 \alpha \pi+\varphi_{6} \cos \frac{3 \alpha \pi}{2}+\varphi_{7} \sin \frac{3 \alpha \pi}{2}+\varphi_{8} \cos \alpha \pi \\
& +\varphi_{9} \sin \alpha \pi+\varphi_{10} \cos \frac{\alpha \pi}{2}+\varphi_{11} \sin \frac{\alpha \pi}{2}+\varphi_{12}, \\
D_{4}= & \omega^{4 \alpha} \sin 2 \alpha \pi+\varphi_{6} \sin \frac{3 \alpha \pi}{2}-\varphi_{7} \cos \frac{3 \alpha \pi}{2}+\varphi_{8} \sin \alpha \pi \\
& -\varphi_{9} \cos \alpha \pi+\varphi_{10} \sin \frac{\alpha \pi}{2}-\varphi_{11} \cos \frac{\alpha \pi}{2}-\varphi_{13},
\end{aligned}
$$

and $\varphi_{i}, i=1,2, \ldots, 13$ are defined by Appendix B.

It follows from (40) that

$$
\left\{\begin{array}{l}
\sin \omega \tau=\frac{D_{1} D_{4}-D_{2} D_{3}}{D_{1}^{2}+D_{2}^{2}}, \\
\cos \omega \tau=-\frac{D_{1} D_{3}+D_{2} D_{4}}{D_{1}^{2}+D_{2}^{2}} .
\end{array}\right.
$$

Similarly, we can obtain

$$
D_{3}^{2}+D_{4}^{2}=D_{1}^{2}+D_{2}^{2}
$$

Assume that equation (40) has at least one positive real root $\omega_{1}$; then, it follows from the second equation of (39) that

$$
\tau_{\delta}^{(i)}=\frac{1}{\omega_{1}}\left[\arccos \frac{D_{1} D_{3}+D_{2} D_{4}}{D_{1}^{2}+D_{2}^{2}}+2 i \pi\right], \quad i=0,1,2, \ldots
$$

Define

$$
\tau_{\delta}^{(i)}=\min \left\{\tau_{\delta}^{(i)}\right\}, \quad i=0,1,2, \ldots
$$

To derive conditions for the existence of Hopf bifurcation, we make the following hypothesis:

$$
\left(H_{4}\right): \frac{\Phi_{1} \Psi_{1}+\Phi_{2} \Psi_{2}}{\Psi_{1}^{2}+\Psi_{2}^{2}} \neq 0
$$

where $\widetilde{\Phi}_{1}, \widetilde{\Phi}_{2}, \widetilde{\Psi}_{1}$, and $\widetilde{\Psi}_{2}$ are defined by equation (47).

Lemma 2. Let $s(\tau)=\varphi(\tau)+i \omega(\tau)$ be a root of the characteristic equation (34) near $\tau=\tau_{\delta}^{(i)}$ meeting $\varphi\left(\tau_{\delta}^{(i)}\right)=0, \omega\left(\tau_{\delta}^{(i)}\right)=\omega_{1}$; then, the transversality condition

$$
\left.\operatorname{Re}\left[\frac{\mathrm{d} s}{\mathrm{~d} \tau}\right]\right|_{\left(\tau=\tau_{\delta}^{0}, \omega=\omega_{1}\right)} \neq 0,
$$

holds.

Proof. Differentiating both sides of (39) with respect to $\tau$, we obtain

$$
\frac{\mathrm{d} s}{\mathrm{~d} \tau}=\frac{\widetilde{\Phi}(s)}{\widetilde{\Psi}(s)},
$$

where $\widetilde{\Phi}(s)=\left(B_{5} s^{2 \alpha}+B_{6} s^{\alpha}+B_{7}\right) s e^{-s \tau}$ and

$$
\begin{aligned}
\widetilde{\Psi}(s)= & 4 \alpha s^{4 \alpha-1}+3 \alpha B_{1} s^{3 \alpha-1}+2 \alpha B_{2} s^{2 \alpha-1}+\alpha B_{3} s^{\alpha-1} \\
& +\left[2 \alpha B_{5} s^{2 \alpha-1}+\alpha B_{6} s^{\alpha-1}-\left(B_{5} s^{2 \alpha}+B_{6} s^{\alpha}+B_{7}\right) \tau\right] e^{-s \tau} \\
& -k \delta e^{-s \delta}\left\{s^{3 \alpha}+\left(m_{3}+\eta\right) s^{2 \alpha}+\left(m_{6}+m_{3} \eta-a b P_{S}^{*}\right) s^{\alpha}\right. \\
& \left.+\eta\left(m_{6}-a b P_{S}^{*}\right)+\left[\beta n P_{I}^{*} s^{\alpha}+\left(m_{2} n P_{I}^{*}+a d P_{S}^{*} V^{*}\right) \beta\right] e^{-s \tau}\right\} .
\end{aligned}
$$

Let

$$
\begin{aligned}
& \left.\widetilde{\Phi}\left(\omega_{1} i\right)\right|_{\tau=\tau_{\delta}^{0}}=\widetilde{\Phi}_{1}+i \widetilde{\Phi}_{2}, \\
& \left.\Psi\left(\omega_{1} i\right)\right|_{\tau=\tau_{\delta}^{0}}=\widetilde{\Psi}_{1}+i \widetilde{\Psi}_{2},
\end{aligned}
$$

where $\widetilde{\Phi}_{1}$ and $\widetilde{\Phi}_{2}$ are the real and imaginary parts of $\widetilde{\Phi}\left(\omega_{1} i\right)$, respectively, while $\widetilde{\Psi}_{1}$ and $\widetilde{\Psi}_{2}$ are the real and imaginary parts of $\widetilde{\Psi}\left(\omega_{1} i\right)$, respectively. 
TABLE 2: Model parameter values from [3, 39-41].

\begin{tabular}{lcc}
\hline Parameter & Value & Unit \\
\hline$r$ & 0.05 & day $^{-1}$ \\
$a$ & 0.005 & kg vector $^{-1}$ day $^{-1}$ \\
$n$ & 0.005 & day $^{-1}$ \\
$c$ & 0.12 & day $^{-1}$ day $^{-1}$ \\
$\gamma$ & 0.03 & day $^{-1}$ \\
$\eta$ & 0.015 & kg.plant $^{-1}$ \\
$K$ & 50 & day $^{-1}$ day $^{-1}$ \\
$M$ & 0.03 & day $^{-1}$ \\
$d$ & 0.8 & day $^{-1}$ \\
$\beta$
\end{tabular}

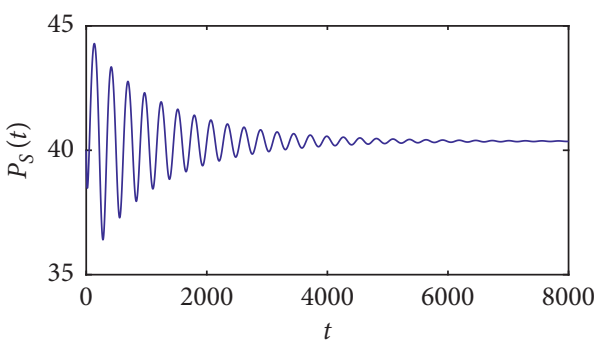

(a)

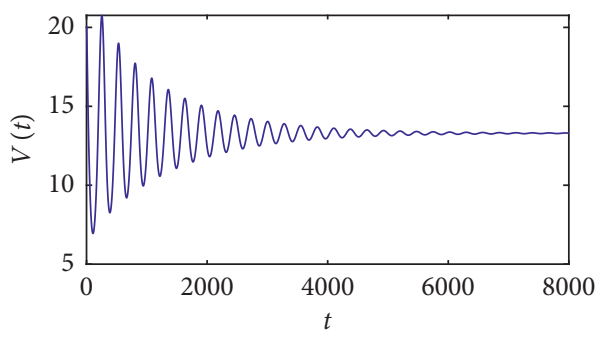

(c)

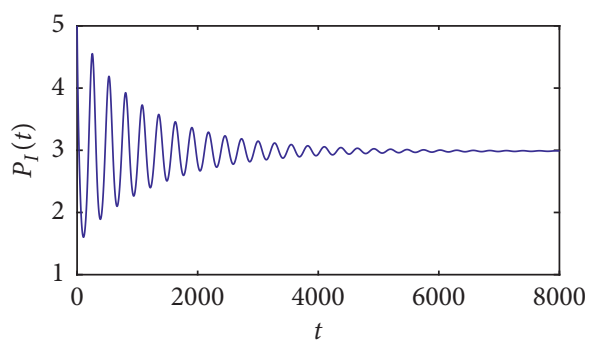

(b)

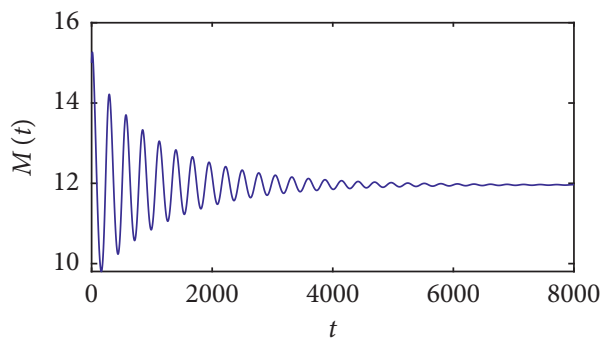

(d)

FIgURE 1: The time series of system (3) when $\alpha=0.95$ and $\tau=45<\tau^{0}$.

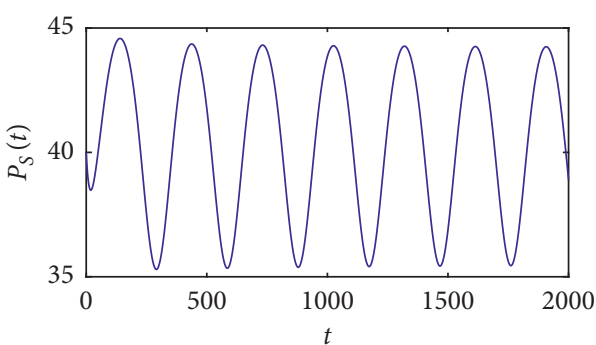

(a)

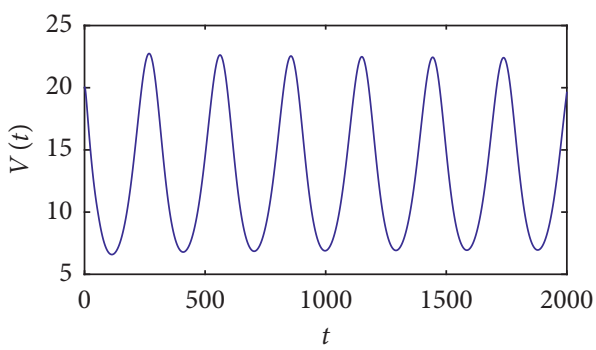

(c)

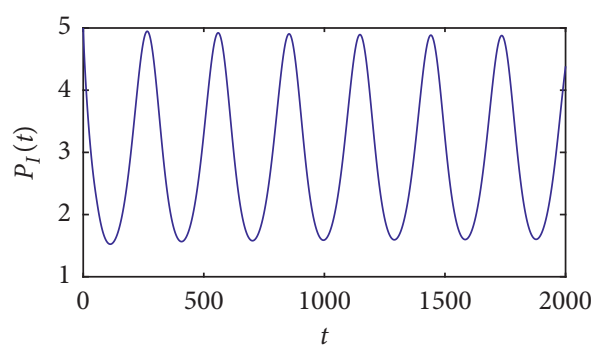

(b)

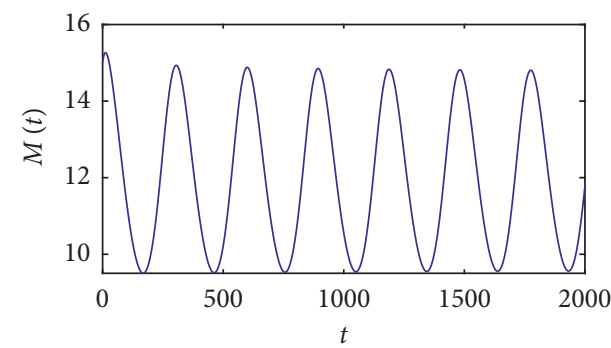

(d)

FIgURe 2: The time series of system (3) when $\alpha=0.95$ and $\tau=53>\tau^{0}$. 


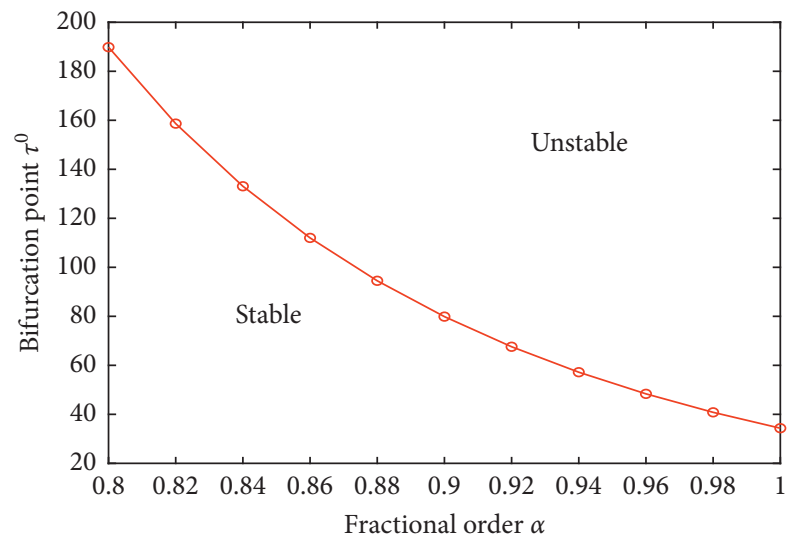

FIgURE 3: The impact of the fractional order $\alpha$ on the bifurcation value $\tau^{0}$ for the uncontrolled system (3).
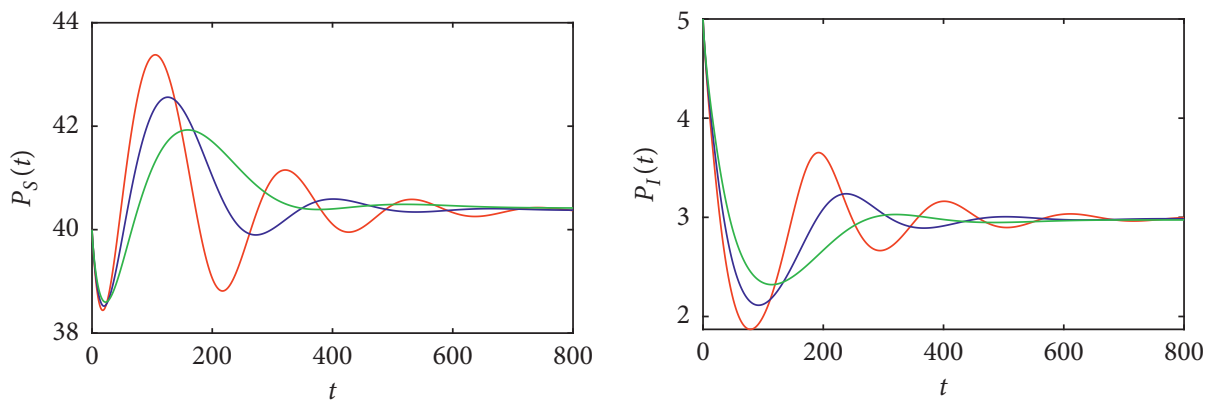

$$
\begin{aligned}
\alpha & =0.98 \\
-\alpha & =0.92 \\
\alpha & =0.86
\end{aligned}
$$$$
-\alpha=0.98
$$$$
-\alpha=0.92
$$

$\alpha=0.86$

(a)

(b)
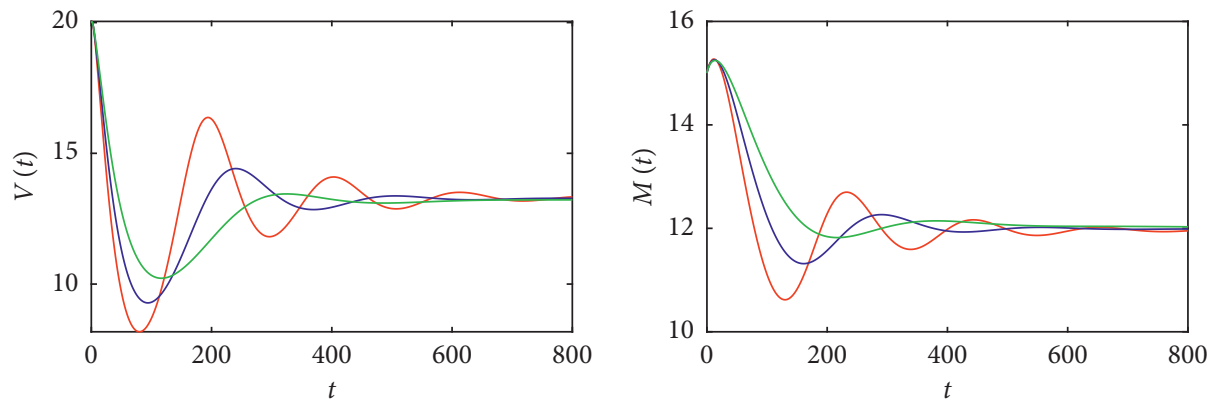

$$
\begin{aligned}
\alpha & =0.98 \\
\alpha & =0.92
\end{aligned}
$$$$
\alpha=0.98
$$$$
-\alpha=0.92
$$$$
\alpha=0.86
$$

(c)

(d)

Figure 4: The time series of system (3) when $\alpha=0.98, \alpha=0.92, \alpha=0.86$, and $\tau=15$.

By straightforward computation, it can be derived from (45) that

$$
\left.\operatorname{Re}\left[\frac{\mathrm{d} s}{\mathrm{~d} \tau}\right]\right|_{\left(\tau=\tau_{\delta}^{0}, \omega=\omega_{1}\right)}=\frac{\widetilde{\Phi}_{1} \widetilde{\Psi}_{1}+\widetilde{\Phi}_{2} \widetilde{\Psi}_{2}}{\widetilde{\Psi}_{1}^{2}+\widetilde{\Psi}_{2}^{2}} \neq 0 .
$$

The proof is completed.
Theorem 2. Suppose assumptions $\left(H_{1}\right),\left(H_{2}\right)$, and $\left(H_{5}\right)$ hold; then,

(1) The coexistence equilibrium $E^{*}$ of system (5) is locally asymptotically stable for $\tau \in\left[0, \tau_{\delta}^{0}\right)$

(2) System (5) undergoes Hopf bifurcation at $E^{*}$ when $\tau=\tau_{\delta}^{0}$ 


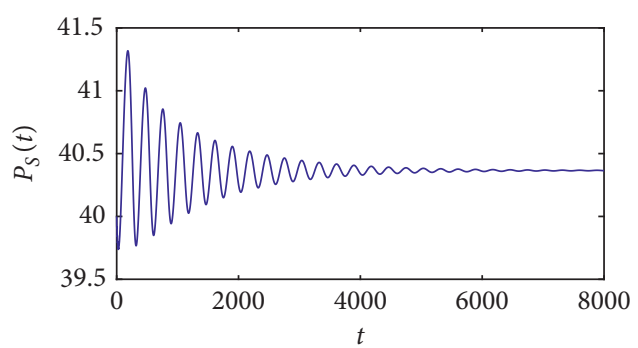

(a)

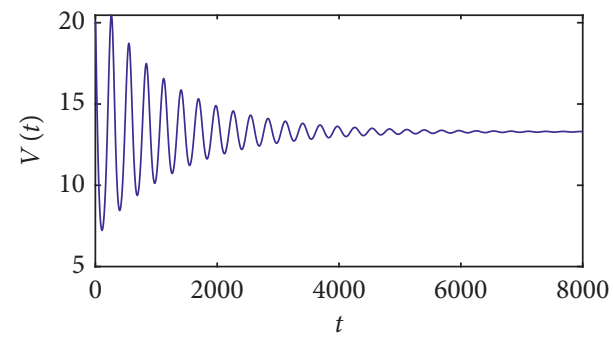

(c)

Figure 5: The time series of system (5) when $\alpha=0.95$ and $\tau=28<\tau_{\delta}^{0}$.

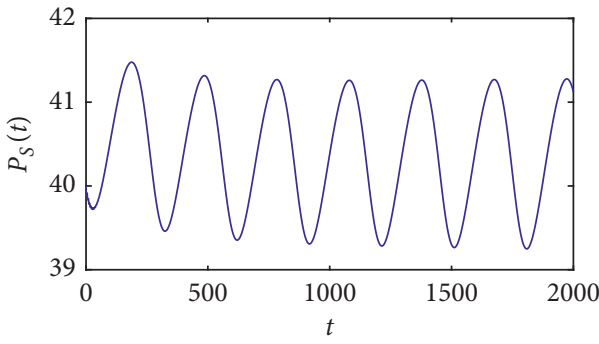

(a)

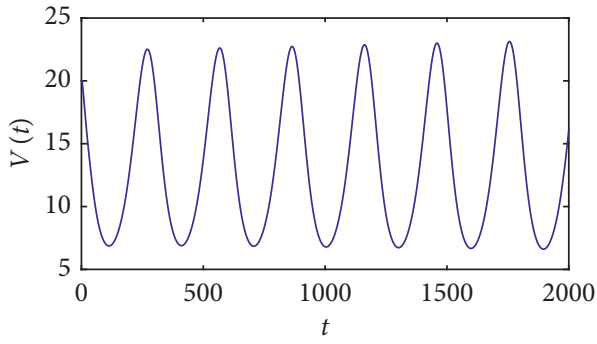

(c)

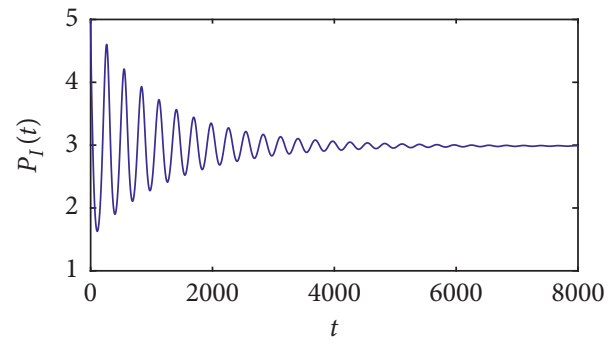

(b)

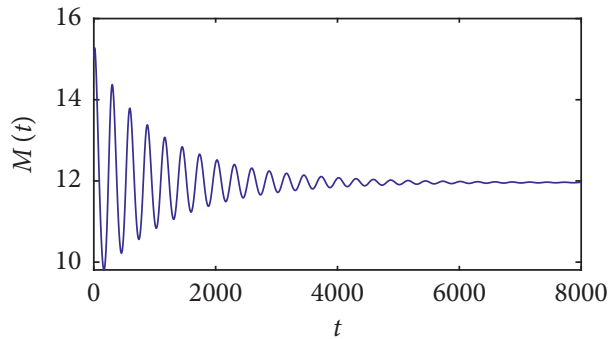

(d)

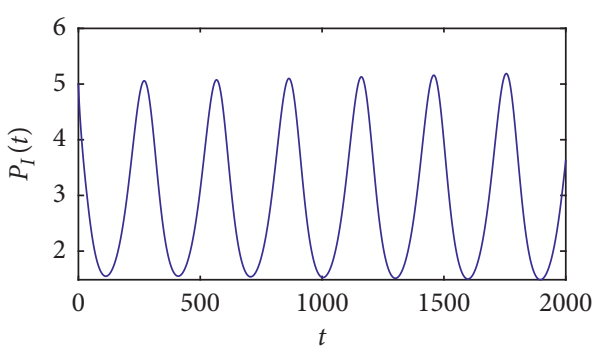

(b)

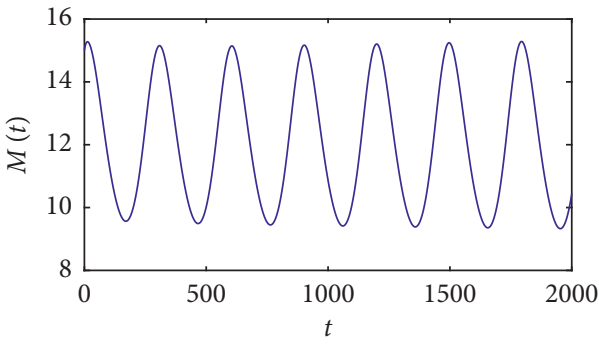

(d)

Figure 6: The time series of system (5) when $\alpha=0.95$ and $\tau=33>\tau_{\delta}^{0}$.

\section{Numerical Simulation}

In this section, two numerical examples are provided to verify our theoretical results of systems (3) and (5).

5.1. Example 1. In the following, we will investigate the stability of the coexistence equilibrium $E^{*}$ and the existence of Hopf bifurcation for system (3). Model parameters are chosen from [3, 39-41] (refer to Table 2).

By simple computation, we get the unique coexistence equilibrium:

$$
E^{*}=(39.3954,2.9012,13.0133,11.6706) \text {. }
$$

Selecting the fractional order as $\alpha=0.95$, we easily obtain that $\omega_{0}=0.0215$ and the critical value $\tau^{0}=52.5989$. According to Theorem 1, the coexistence equilibrium $E^{*}$ is asymptotically stable when $\tau=45<\tau^{0}$, which is shown in Figure 1. Figure 2 shows that the coexistence equilibrium $E^{*}$ is unstable when $\tau=53>\tau^{0}$ and a Hopf bifurcation occurs.

In addition, we explore the impact of the fractional order $\alpha$ on the bifurcation value $\tau^{0}$ for the uncontrolled system (3). 


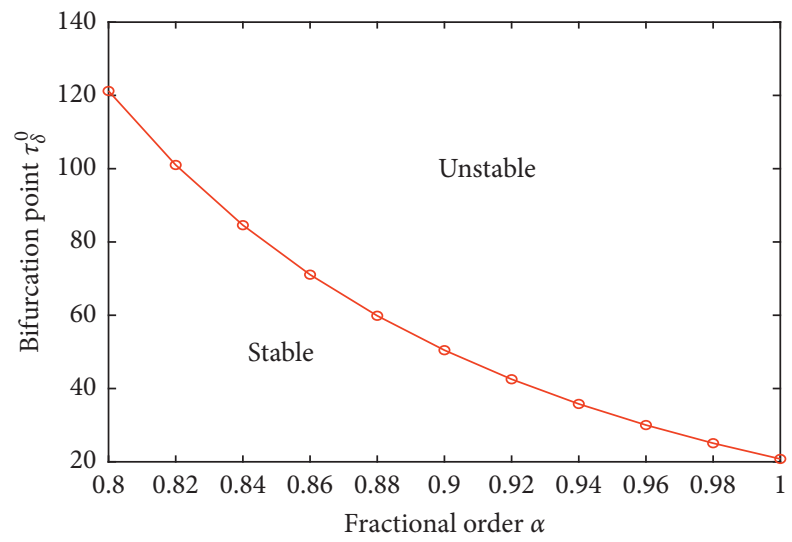

Figure 7: The impact of the fractional order $\alpha$ on the bifurcation value $\tau_{\delta}^{0}$ for the controlled system (5).

From Figure 3, we can see that the bifurcation value $\tau^{0}$ is very integral order, the fractional system has a larger stability
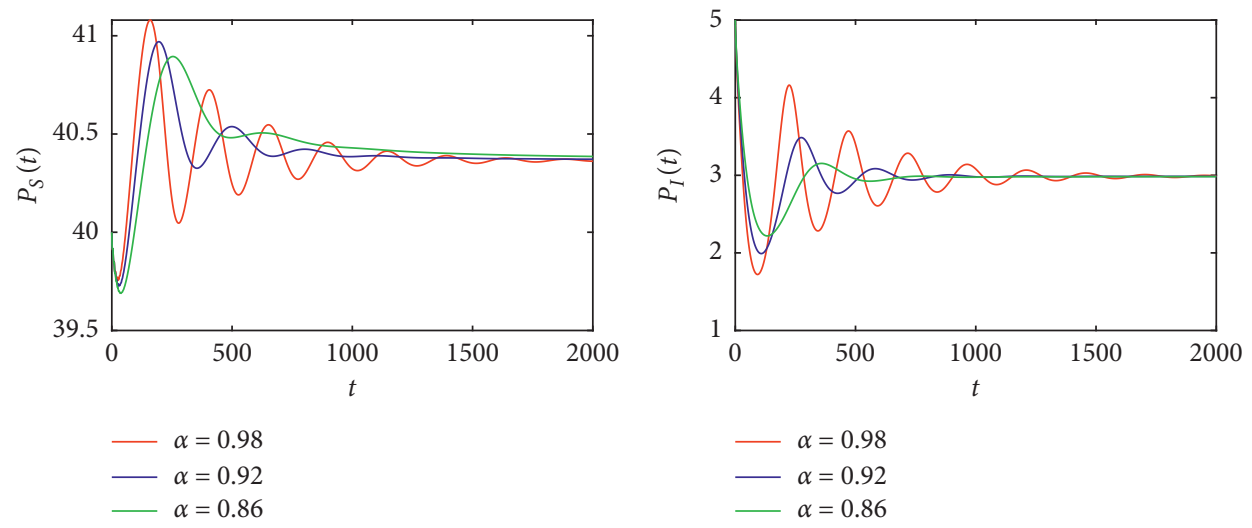

(a)
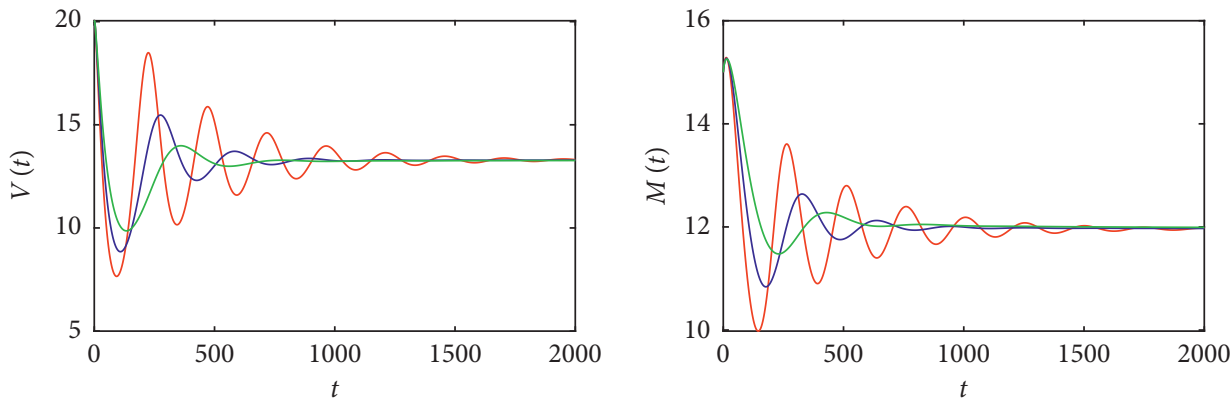

$\begin{aligned} \alpha & =0.98 \\ -\alpha & =0.92 \\ \alpha & =0.86\end{aligned}$

$\begin{aligned} \alpha & =0.98 \\ -\alpha & =0.92 \\ \alpha & =0.86\end{aligned}$

(c)

(d)

Figure 8: The time series of system (5) when $\alpha=0.98, \alpha=0.92, \alpha=0.86, k=-2, \delta=4.8$, and $\tau=15$.

sensitive to the change of the fractional order $\alpha$. With the increase of the order, the bifurcation value decreases rapidly, which implies that the stability region of system (3) becomes smaller. In particular, compared to the corresponding region.

Besides, by selecting $\tau=15$, we investigate the convergence rate of the system. From Figure 4, we find that, with the increase of the order $\alpha$, the convergence rate of system (3) becomes slower. 


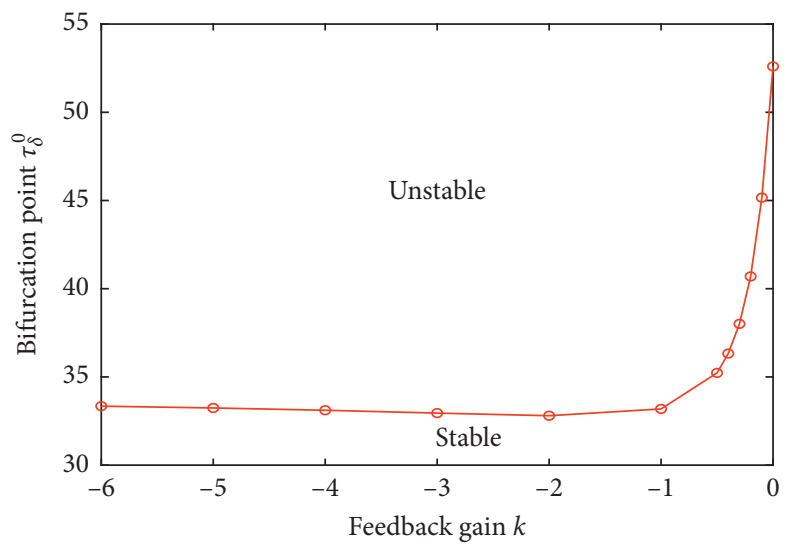

FIgURE 9: The impact of the feedback gain $k$ on the bifurcation value $\tau_{\delta}^{0}$ for the controlled system (5).

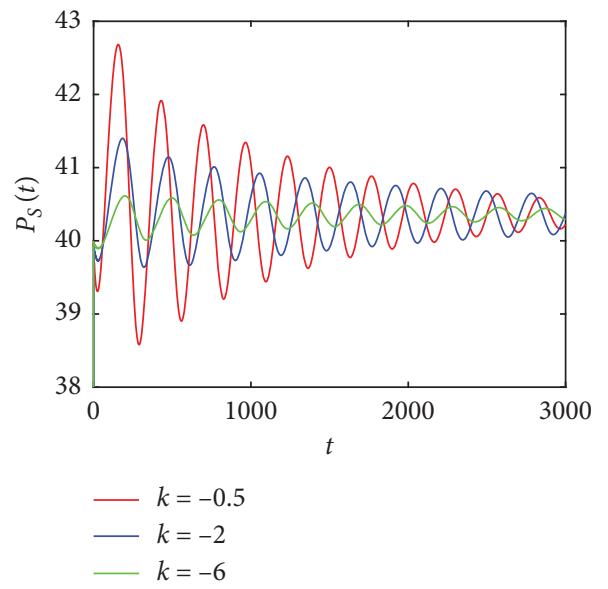

(a)

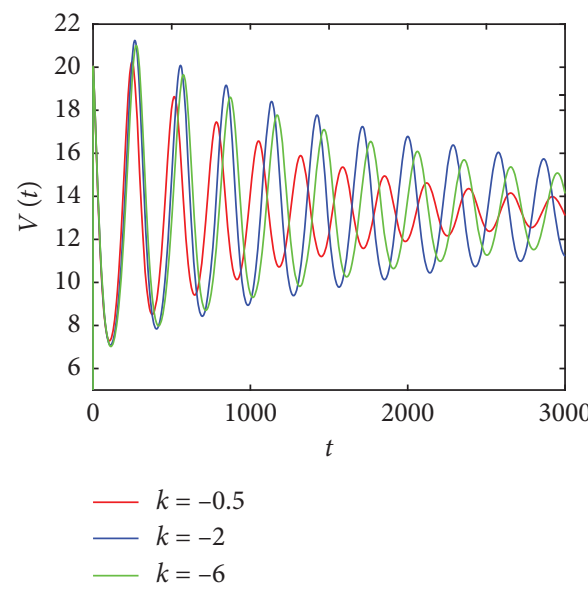

(c)

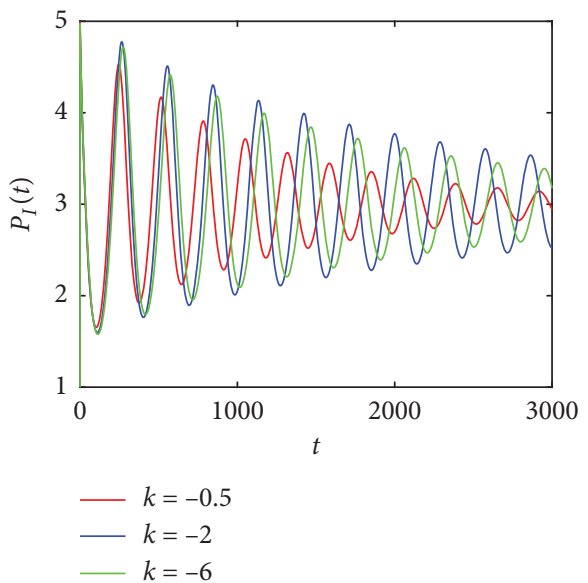

(b)

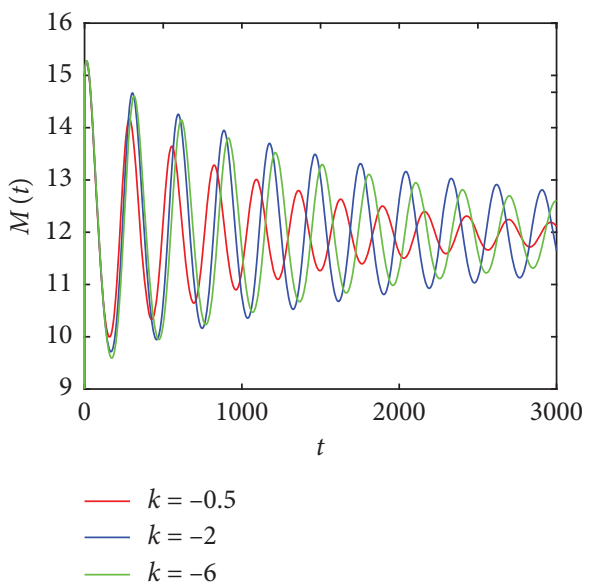

(d)

Figure 10: The time series of system (5) when $\alpha=0.95, k=-0.5, k=-2, k=-6, \delta=4.8$, and $\tau=30$.

5.2. Example 2. Now, we study the bifurcation control problem of uncontrolled system (3) by introducing the time-delayed feedback controller. Model parameters of controlled system (5) are chosen the same as in Table 2, while the feedback gain and the feedback delay are selected as $k=-2$ and $\delta=4.8$, respectively. When the order is chosen as $\alpha=0.95$, we can obtain that $\omega_{1}=0.0213$ and the corresponding critical value $\tau_{\delta}^{0}=32.8007$. According to Theorem 2, the coexistence equilibrium $E^{*}$ is asymptotically stable when $\tau=28<\tau_{\delta}^{0}$ (see Figure 5), while it is unstable when $\tau=33>\tau_{\delta}^{0}$ and a Hopf bifurcation occurs (see Figure 6). 


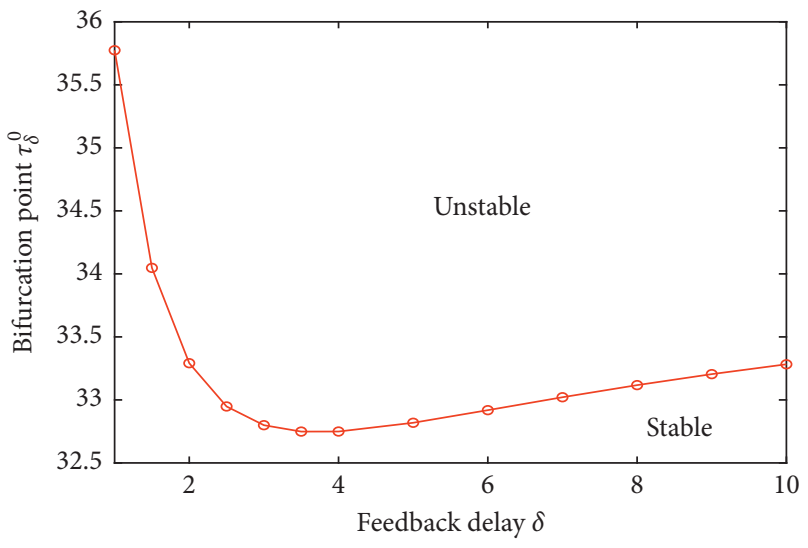

FIGURE 11: The impact of the extended feedback delay $\delta$ on the bifurcation value $\tau_{\delta}^{0}$ for the controlled system (5).

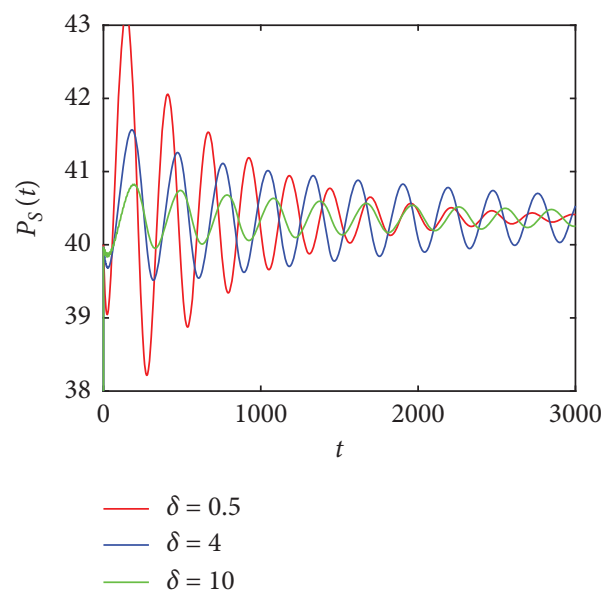

(a)

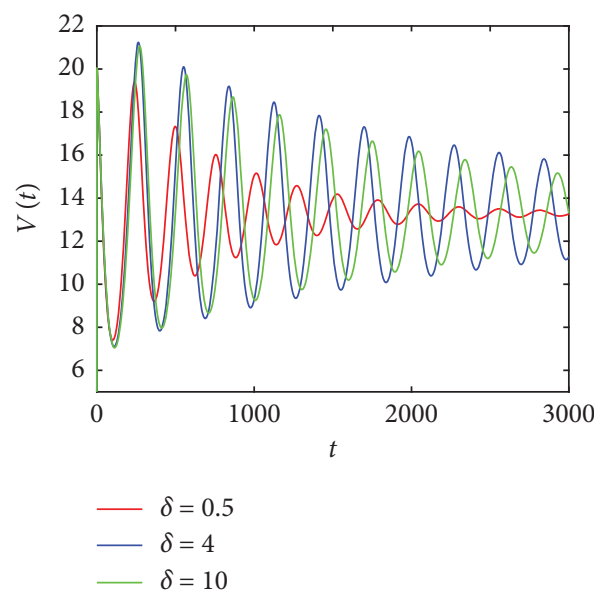

(c)

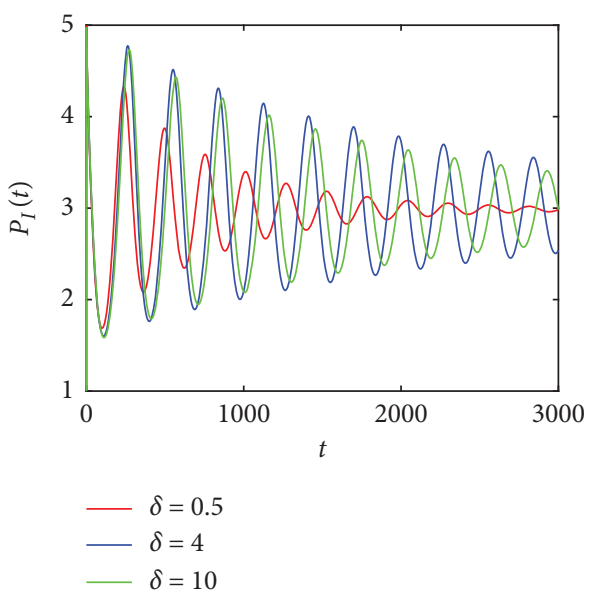

(b)

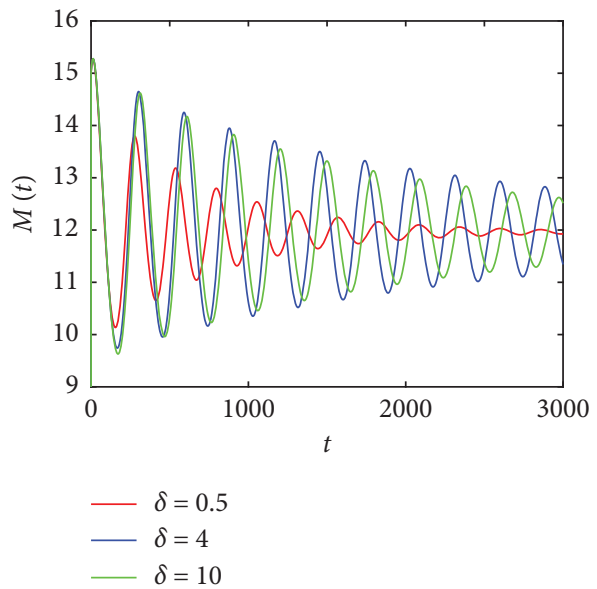

(d)

Figure 12: The time series of system (5) when $\alpha=0.98, k=-2, \delta=0.5, \delta=4, \delta=10$, and $\tau=30$.

We also explore the impact of the fractional order $\alpha$ on the bifurcation value $\tau_{\delta}^{0}$ for the controlled system (5). From Figure 7 , we can see that the bifurcation value decreases rapidly with the increase of the order, which means that the stability region of system (5) also becomes smaller. Similarly, with the rise of the order $\alpha$, the convergence rate of system (5) slows down (see Figure 8).

Furthermore, we investigate the impact of the feedback gain $k$ and the feedback delay $\delta$ on the bifurcation value $\tau_{\delta}^{0}$. By fixing $\alpha=0.95, \delta=4.8$, and $\tau=30$, we find that there is a 
minimum point around $k=-2$ (see Figure 9). Specifically, with the increase of the feedback gain $k$, the stability region of system (5) first slowly reduces to a minimum, then gradually increases to a high level. Three representative values $k=-0.5, k=-2$, and $k=-6$ are chosen in Figure 10, and we can see that system (5) displays the slowest convergence rate when $k=-2$ (which is the nearest to the minimum point).

In addition, by fixing $\alpha=0.95, k=-2$, and $\tau=30$, we can see from Figure 11 that there is also a minimum point around $\delta=3.5$. Specifically, with the increase of the feedback delay $\delta$, the stability region of system (5) first quickly reduces to a minimum and then slowly increases. Three representative values $\delta=0.5, \delta=4$, and $\delta=10$ are chosen, and we can see that system (5) converges the slowest when $\delta=4$ which is the nearest to the minimum point (see Figure 12).

\section{Conclusion}

In this paper, a fractional-order mosaic virus infection model for Jatropha curcas with farming awareness and an execution delay was studied based on the model in [2]. Compared with the studies, we not only generalized the integral order system to a fractional-order system which may describe the mechanism of the disease transmission more accurately but also investigated the Hopf bifurcation control through a time-delayed feedback controller. We mainly studied the existence of Hopf bifurcation and explored the impact of time delay, the fractional order, the feedback gain, and the feedback delay on the occurrence of bifurcation phenomenon.

By choosing the execution delay as a bifurcation parameter, Hopf bifurcation of the uncontrolled system was firstly studied. The stability of the coexistence equilibrium and the bifurcation criteria were obtained. Then, by introducing a time-delayed feedback controller, the bifurcation control problem of the uncontrolled system was investigated in detail. A series of numerical simulations were performed, which not only verified our theoretical results but also revealed some specific features. Numerically, we exploited the impact of the time delay, the fractional order, the feedback gain, and the feedback delay on the occurrence of Hopf bifurcation. We found that, for both uncontrolled and controlled systems, when the execution delay is small enough (less than the critical values), the systems are stable, while they lose their stability and Hopf bifurcations occur when the execution delay exceeds the critical values. Besides, our study showed that the bifurcation values are very sensitive to the change of the fractional order. With the increase of the order, the bifurcation values of the two systems decrease rapidly, which implies that the stability region of systems becomes smaller. Furthermore, we investigated the impact of the feedback gain and the extended feedback delay on the bifurcation value for the controlled system, and we found the existence of the extreme points for the feedback gain and the extended feedback delay which can minimize the bifurcation value.
According to our study, the execution time delay can lead to the fluctuation of the population quantity of Jatropha plants, which means that the population quantity is at some unreasonable level. By introducing a timedelayed feedback controller, we can stabilize the population by harvesting or transplanting new plants according to the current and past population level (at time $t$ and $t-\delta$ ). If the population quantity of the healthy plant at $t-\delta$ is more than the current level, we harvest some plants. Otherwise, we transplant some new plants. Our numerical simulations show that there exist extreme points for the feedback gain $k$ and the feedback delay $\delta$ which can both minimize the bifurcation value, so we can select values of the parameters $k$ and $\delta$ which are far away from those extreme points, then the population size tends to be stabilized. Specifically, we prefer to choose the feedback gain $k$ which is greater than the minimum point and choose the feedback delay $\delta$ which is less than the minimum point because these values are more conducive to a stable population level.

For the control of system (3), we can also add a controller to the other equations or add more than one controller at a time, and comparisons between different controllers would be very meaningful. Besides, introducing artificial impulsive control behaviors into system (3) is also interesting. We will continue these studies in our future work.

\section{Appendix}

\section{A. Important Expressions}

The expressions of $n_{i}, i=1,2, \ldots, 6$ in (31):

$$
\begin{aligned}
n_{1}= & A_{5} \omega_{0}^{2 \alpha+1} \cos \alpha \pi+A_{6} \omega_{0}^{\alpha+1} \cos \frac{\alpha \pi}{2}+A_{7} \omega_{0} \\
n_{2}= & A_{5} \omega_{0}^{2 \alpha+1} \sin \alpha \pi+A_{6} \omega_{0}^{\alpha+1} \sin \frac{\alpha \pi}{2} \\
n_{3}= & 4 \alpha \omega_{0}^{4 \alpha-1} \cos \frac{(4 \alpha-1) \pi}{2}+3 \alpha A_{1} \omega_{0}^{3 \alpha-1} \cos \frac{(3 \alpha-1) \pi}{2} \\
& +2 \alpha A_{2} \omega_{0}^{2 \alpha-1} \cos \frac{(2 \alpha-1) \pi}{2}+\alpha A_{3} \omega_{0}^{\alpha-1} \cos \frac{(\alpha-1) \pi}{2} \\
n_{4}= & 4 \alpha \omega_{0}^{4 \alpha-1} \sin \frac{(4 \alpha-1) \pi}{2}+3 \alpha A_{1} \omega_{0}^{3 \alpha-1} \sin \frac{(3 \alpha-1) \pi}{2} \\
& +2 \alpha A_{2} \omega_{0}^{2 \alpha-1} \sin \frac{(2 \alpha-1) \pi}{2}+\alpha A_{3} \omega_{0}^{\alpha-1} \sin \frac{(\alpha-1) \pi}{2} \\
n_{5}= & 2 \alpha A_{5} \omega_{0}^{2 \alpha-1} \cos \frac{(2 \alpha-1) \pi}{2}+\alpha A_{6} \omega_{0}^{\alpha-1} \cos \frac{(\alpha-1) \pi}{2} \\
& -A_{5} \tau^{0} \omega_{0}^{2 \alpha} \cos \alpha \pi-A_{6} \tau^{0} \omega_{0}^{\alpha} \cos \frac{\alpha \pi}{2}-A_{7} \tau^{0}, \\
n_{6}= & 2 \alpha A_{5} \omega_{0}^{2 \alpha-1} \sin \frac{(2 \alpha-1) \pi}{2}+\alpha A_{6} \omega_{0}^{\alpha-1} \sin \frac{(\alpha-1) \pi}{2} \\
& -A_{5} \tau^{0} \omega_{0}^{2 \alpha} \sin \alpha \pi-A_{6} \tau^{0} \omega_{0}^{\alpha} \sin \frac{\alpha \pi}{2} .
\end{aligned}
$$




\section{B. Important Expressions}

The expressions of $\varphi_{i}, i=1,2, \ldots, 13$ in (37):

$$
\begin{aligned}
\varphi_{1} & =A_{5} \omega^{2 \alpha}, \\
\varphi_{2} & =\left[A_{6}-\beta n P_{I}^{*} k(1-\cos \delta \omega)\right] \omega^{\alpha}, \\
\varphi_{3} & =\beta n P_{I}^{*} k \omega^{\alpha} \sin \delta \omega, \\
\varphi_{4} & =A_{7}-\left(m_{2} n P_{I}^{*}+a d P_{S}^{*} V^{*}\right) \beta k(1-\cos \delta \omega), \\
\varphi_{5} & =\left(m_{2} n P_{I}^{*}+a d P_{S}^{*} V^{*}\right) \beta k \sin \delta \omega, \\
\varphi_{6} & =\left[A_{1}-k(1-\cos \delta \omega)\right] \omega^{3 \alpha}, \\
\varphi_{7} & =k \omega^{3 \alpha} \sin \delta \omega, \\
\varphi_{8} & =\left[A_{2}-\left(m_{3}+\eta\right) k(1-\cos \delta \omega)\right] \omega^{2 \alpha}, \\
\varphi_{9} & =\left(m_{3}+\eta\right) k \omega^{2 \alpha} \sin \delta \omega, \\
\varphi_{10} & =\left[A_{3}-\left(m_{6}+m_{3} \eta-a b P_{S}^{*}\right) k(1-\cos \delta \omega)\right] \omega^{\alpha}, \\
\varphi_{11} & =\left(m_{6}+m_{3} \eta-a b P_{S}^{*}\right) k \omega^{\alpha} \sin \delta \omega, \\
\varphi_{12} & =A_{4}-\eta\left(m_{6}-a b P_{S}^{*}\right) k(1-\cos \delta \omega), \\
\varphi_{13} & =\eta\left(m_{6}-a b P_{S}^{*}\right) k \sin \delta \omega .
\end{aligned}
$$

\section{Data Availability}

The data used to support the findings of this study are included within the article.

\section{Conflicts of Interest}

The authors declare that they have no conflicts of interest.

\section{Acknowledgments}

This work was supported by the National Natural Science Foundation of China (11901502, 11671346, and 11871415) and Training plan for young backbone teachers in Henan Province (2019GGJS157), Nanhu Scholars Program for Young Scholars of XYNU.

\section{References}

[1] P. K. Roy, S. Datta, S. Nandi, and F. Al Basir, "Effect of mass transfer kinetics for maximum production of biodiesel from Jatropha curcas oil: a mathematical approach," Fuel, vol. 134, pp. 39-44, 2014.

[2] F. A. Basir, E. Venturino, S. Ray, and P. K. Roy, "Impact of farming awareness and delay on the dynamics of mosaic disease in Jatropha curcas plantations," Computainal and Applied Mathematics, vol. 37, no. 3, pp. 6108-6131, 2018.

[3] F. A. Basir, E. Venturino, and P. K. Roy, "Effects of awareness program for controlling mosaic disease in Jatropha curcas plantations," Mathematical Methods in the Applied Sciences, vol. 40, no. 7, pp. 2441-2453, 2017.

[4] E. Venturino, P. K. Roy, F. A. Basir, and D. Abhirup, “A model for the control of the mosaic virus disease in Jatropha curcas plantations," Energy, Ecology and Environment, vol. 1, no. 6, pp. 360-369, 2016.

[5] R. Ramkat, A. Calari, F. Maghuly, and M. Laimer, "Occurrence of African cassava mosaic virus (ACMV) and east
African cassava mosaic virus- Uganda (EACMV-UG) in Jatropha curcas," BMC Proceedings, vol. 5, no. 7, p. 93, 2011.

[6] S. K. Raj, S. K. Snehi, S. Kumar, M. S. Khan, and U. Pathre, "First molecular identification of a begomovirus in India that is closely related to cassava mosaic virus and causes mosaic and stunting of Jatropha curcas L." Australasian Plant Disease Notes, vol. 3, no. 1, pp. 69-72, 2008.

[7] G. A. Khan, S. Muhammad, and M. A. Khan, "Information regarding agronomic practices and plant protection measures obtained by the farmers through electronic media," Journal of Animal and Plant Sciences, vol. 23, no. 2, pp. 647-650, 2013.

[8] L. Klerkx, N. Aarts, and C. Leeuwis, "Adaptive management in agricultural innovation systems: the interactions between innovation networks and their environment," Agricultural Systems, vol. 103, no. 6, pp. 390-400, 2010.

[9] M. Schut, J. Rodenburg,L. Klerkx, A. van Ast, and L. Bastiaans, "Systems approaches to innovation in crop protection. A systematic literature review," Crop Protection, vol. 56, pp. 98-108, 2014.

[10] X. Yang, F. Wang, L. Meng et al., "Farmer and retailer knowledge and awareness of the risks from pesticide use: a case study in the Wei river catchment, China," Science of the Total Environment, vol. 497-498, no. 1, pp. 172-179, 2014.

[11] F. Le Bellec, A. Rajaud, H. Ozier-Lafontaine, C. Bockstaller, and E. Malezieux, "Evidence for farmers' active involvement in co-designing citrus cropping systems using an improved participatory method," Agronomy for Sustainable Development, vol. 32, no. 3, pp. 703-714, 2012.

[12] F. A. Basir, A. Banerjee, and S. Ray, "Role of farming awareness in crop pest management-a mathematical model," Journal of Theoretical Biology, vol. 461, pp. 59-67, 2019.

[13] R. P. Agarwal, M. Benchohra, and S. Hamani, "A survey on existence results for boundary value problems of nonlinear fractional differential equations and inclusions," Acta Applicandae Mathematicae, vol. 109, no. 3, pp. 973-1033, 2010.

[14] J. Alidousti and R. K. Ghaziani, "Spiking and bursting of a fractional order of the modified FitzHugh-Nagumo neuron model," Mathematical Models and Computer Simulations, vol. 9, no. 3, pp. 390-403, 2017.

[15] J. Alidousti and M. Mostafavi Ghahfarokhi, "Dynamical behavior of a fractional three-species food chain model," Nonlinear Dynamics, vol. 95, no. 3, pp. 1841-1858, 2019.

[16] N. Laskin and G. Zaslavsky, "Nonlinear fractional dynamics on a lattice with long range interactions," Physica A: Statistical Mechanics and Its Applications, vol. 368, no. 1, pp. 38-54, 2006.

[17] T. J. Anastasio, "The fractional-order dynamics of brainstem vestibulo-oculomotor neurons," Biological Cybernetics, vol. 72, no. 1, pp. 69-79, 1994.

[18] W.-C. Chen, "Nonlinear dynamics and chaos in a fractionalorder financial system," Chaos, Solitons \& Fractals, vol. 36, no. 5, pp. 1305-1314, 2008.

[19] W. Lin, "Global existence theory and chaos control of fractional differential equations," Journal of Mathematical Analysis and Applications, vol. 332, no. 1, pp. 709-726, 2007.

[20] T. A. Yildiz, "A fractional dynamical model for honeybee colony population," International Journal of Biomathematics, vol. 11, no. 5, Article ID 18500638, 2018.

[21] J. E. Escalante-Martnez, J. F. Gmez-Aguilar, C. CaldernRamn, A. Aguilar-Meléndez, and P. Padilla-Longoria, "A mathematical model of circadian rhythms synchronization using fractional differential equations system of coupled van der pol oscillators," International Journal of Biomathematics, vol. 11, no. 1, Article ID 1850014, 2018. 
[22] M. Xiao, W. X. Zheng, J. Lin, G. Jiang, L. Zhao, and J. Cao, "Fractional-order PD control at Hopf bifurcations in delayed fractional-order small-world networks," Journal of the Franklin Institute, vol. 354, no. 17, pp. 7643-7667, 2017.

[23] C. Xu, M. Liao, P. Li, Q. Xiao, and S. Yuan, "PD" control strategy for a fractional-order chaotic financial model," Complexity, vol. 2019, Article ID 2989204, 14 pages, 2019.

[24] C. Huang, J. Cao, M. Xiao, A. Alsaedi, and T. Hayat, "Effects of time delays on stability and Hopf bifurcation in a fractional ring-structured network with arbitrary neurons," Communications in Nonlinear Science and Numerical Simulation, vol. 57, pp. 1-13, 2018.

[25] C. Xu, M. Liao, P. Li, Y. Guo, Q. Xiao, and S. Yuan, "Influence of multiple time delays on bifurcation of fractional-order neural networks," Applied Mathematics and Computation, vol. 361, pp. 565-582, 2019.

[26] C. Xu, P. Li, M. Liao, and S. Yuan, "Bifurcation analysis for a fractional-order chemotherapy model with two different delays," Mathematical Methods in the Applied Sciences, vol. 43, no. 3, pp. 1053-1083, 2020.

[27] A. S. Deshpande, V. Daftardar-Gejji, and Y. V. Sukale, "On Hopf bifurcation in fractional dynamical systems," Chaos, Solitons \& Fractals, vol. 98, pp. 189-198, 2017.

[28] C. Xu, P. Li, M. Liao, Z. Liu, Q. Xiao, and S. Yuan, "Control scheme for a fractional-order chaotic Genesio-Tesi model," Complexity, vol. 2019, Article ID 4678394, 15 pages, 2019.

[29] Z. Wang, Y. Xie, J. Lu, and Y. Li, "Stability and bifurcation of a delayed generalized fractional-order prey-predator model with interspecific competition," Applied Mathematics and Computation, vol. 347, pp. 360-369, 2019.

[30] C. Xu, M. Liao, and P. Li, "Bifurcation of a fractional-order delayed malware propagation model in social networks," Discrete Dynamics in Nature and Society, vol. 2019, Article ID 7057052, 10 pages, 2019.

[31] G. Chen, J. L. Moiola, and H. O. Wang, "Bifurcation control: theories, methods, and applications," International Journal of Bifurcation and Chaos, vol. 10, no. 3, pp. 511-548, 2000.

[32] Q. Lu, M. Xiao, Z. Cheng, Y. Song, C. Huang, and J. Cao, "Stability and bifurcation analysis of a fractional-order singlegene regulatory model with delays under a novel $\mathrm{PD}^{\alpha}$ control law," International Journal of Biomathematics, vol. 13, no. 3, Article ID 2050016, 2020.

[33] C. Huang, J. Cao, and M. Xiao, "Hybrid control on bifurcation for a delayed fractional gene regulatory network," Chaos, Solitons \& Fractals, vol. 87, pp. 19-29, 2016.

[34] E. Schöll, G. Hiller, P. Hövel, and M. A. Dahlem, "Time-delayed feedback in neurosystems," Philosophical Transactions of the Royal Society A Mathematical Physical and Engineering Sciences, vol. 367, no. 1891, pp. 1079-1096, 2009.

[35] E. C. de Oliveira and J.A. T. Machado, "A review of definitions for fractional derivatives and integral," Theory and Applications of Fractional Order Systems, vol. 2014, Article ID 238459, 6 pages, 2014

[36] I. Podlubny, Fractional Differential Equations, Academic Press, NewYork, NY, USA, 1999.

[37] S. Li, C. Huang, and X. Song, "Bifurcation based-delay feedback control strategy for a fractional-order two-prey onepredator system," Complexity, vol. 2019, Article ID 9673070, 13 pages, 2019.

[38] W. Deng, C. Li, and J. Lü, "Stability analysis of linear fractional differential system with multiple time delays," Nonlinear Dynamics, vol. 48, no. 4, pp. 409-416, 2007.

[39] F. A. Basir and P. K. Roy, "Dynamics of mosaic disease with roguing and delay in Jatropha curcas plantations," Journal of
Applied Mathematics and Computing, vol. 58, no. 1-2, pp. 131, 2018.

[40] J. Holt, M. J. Jeger, J. M. Thresh, and G. W. Otim-Nape, “An epidemilogical model incorporating vector population dynamics applied to african cassava mosaic virus disease," The Journal of Applied Ecology, vol. 34, no. 3, pp. 793-806, 1997.

[41] G. O. Agaba, Y. N. Kyrychko, and K. B. Blyuss, "Time-delayed SIS epidemic model with population awareness," Ecological Complexity, vol. 31, pp. 50-56, 2017. 